\title{
Lipoma intraoral: relato de caso e atualização da literatura
}

\author{
Intraoral lipoma: case report and literature update \\ Lipoma intraoral: reporte de caso y actualización de literature
}

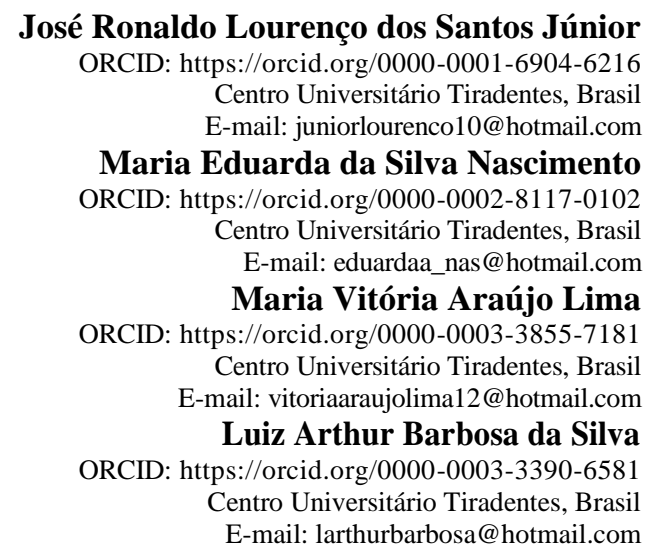

\begin{abstract}
Resumo
O objetivo do presente estudo foi relatar um caso de lipoma diagnosticado em um paciente do sexo masculino, 56 anos de idade, melanoderma e que apresentou lesão nodular. Este processo patológico apresentava-se assintomático, bem delimitado, de consistência amolecida, recoberto por mucosa de superfície íntegra, com coloração amarelada e média, aproximadamente, $2,5 \mathrm{~cm}$. Sua evolução era de aproximadamente 4 anos e estava localizado em fundo de vestíbulo inferior entre os dentes 32 e 34 . Diante dos aspectos clínicos, levantou-se a hipótese diagnóstica de lipoma. Assim, foi realizada a biópsia excisional e, em junção ao exame histopatológico do espécime removido, pôde-se confirmar o diagnóstico clínico da lesão. O paciente encontra-se há 7 meses sem sinais de recidiva da mesma. Vale destacar a necessidade da realização de anamnese criteriosa, além do conhecimento acerca dos achados clínicos pelo Cirurgião-Dentista, o que capacita e, também, proporciona uma correta conduta terapêutica da referida lesão por estes profissionais.
\end{abstract}

Palavras-chave: Lipoma; Diagnóstico; Terapêutica.

\begin{abstract}
The aim of the present study was to report a lipoma case diagnosed in a male, black, 56-year-old patient, who presented with a nodular lesion. This pathological process was asymptomatic, well defined lesion, of softened consistency, covered by mucosa with an intact surface, yellowish in color and measuring approximately $2.5 \mathrm{~cm}$. Its evolution was approximately 4 years and was located in the bottom of the lower vestibule, between teeth 32 and 34 . In view of the clinical aspects, the diagnostic Lipoma hypothesis was raised. Thus, an excisional biopsy was performed and, in addition to the histopathological examination of the removed specimen, it was possible to confirm the clinical diagnosis of the lesion. The patient has had no signs of recurrence for 7 months. It is worth highlighting the need for careful anamnesis, in addition to knowledge about the clinical findings by the Dental Surgeon, which enables and also provides a correct therapeutic approach for this injury by these professionals.
\end{abstract}

Keywords: Lipoma; Diagnosis; Therapeutics.

\section{Resumen}

El objetivo del presente estudio fue reportar un caso de lipoma diagnosticado en un varón de 56 años, de raza negra, que presentaba una lesión nodular. Este proceso patológico fue asintomático, bien delimitado, de consistencia blanda, cubierto de mucosa con una superficie intacta, de color amarillento y de aproximadamente $2,5 \mathrm{~cm}$. Su evolución fue de aproximadamente 4 años y se localizó en el fondo del vestíbulo inferior, entre los dientes 32 y 34 . A la vista de los aspectos clínicos, se planteó la hipótesis diagnóstica de Lipoma. Así, se realizó una biopsia excisional y, además del examen histopatológico de la pieza extraída, se pudo confirmar el diagnóstico clínico de la lesión. El paciente no ha presentado signos de recurrencia durante 7 meses. Cabe destacar la necesidad de una cuidadosa anamnesis, además del conocimiento de los hallazgos clínicos por parte del Cirujano Dentista, que posibilite y además proporcione un correcto abordaje terapéutico de esta lesión por parte de estos profesionales.

Palabras clave: Lipoma; Diagnóstico; Terapéutica. 


\section{Introdução}

O lipoma é uma neoplasia benigna de origem mesenquimal caracterizada pela proliferação de adipócitos maduros. É mais frequente em outras regiões do corpo do que na cavidade oral e, quando comparado às neoplasias benignas comuns nessa região, corresponde a apenas 5\%. Apesar de alguns autores estabelecerem que a obesidade influencia no desenvolvimento do lipoma, acredita-se que a lesão seja completamente independente do metabolismo lipídico corpóreo normal (Osterne et al., 2019; Valencia, Rivera \& Mazuera, 2017). A etiopatogenia dos lipomas, mecanismos de crescimento e manutenção tumoral ainda são desconhecidos, entretanto, fatores inflamatórios, alterações endócrinas, herança genética, trauma local e metaplasia de células do tecido muscular podem estar relacionados ao seu surgimento (Santos et al., 2014; Dehghani et al., 2019).

Clinicamente, apresenta-se como uma lesão nodular de crescimento lento e assintomática, com base séssil ou pediculada, bem circunscrita, consistência macia, superfície lisa e coloração tipicamente amarelada. A região da cavidade oral com maior prevalência é a mucosa jugal, seguida pelo lábio inferior (Osterne et al., 2019; Valencia, Rivera \& Mazuera, 2017). Podem ser incluídas no diagnóstico diferencial lesões como fibroma, hiperplasia fibrosa inflamatória, tumores de glândula salivar, lipossarcoma, cistos dermoides e epidermóides, mucocele e rânula. O processo de diagnóstico é iniciado durante o exame clínico, contudo, é essencial que seja realizada biópsia associada ao exame histopatológico para o estabelecimento do diagnóstico final (Ferreira et al., 2019; Sousa et al., 2008).

Outra característica importante dessa neoplasia é que ao ser colocada no formol a 10\% a mesma flutua devido a sua baixa densidade. Do ponto de vista do histopatológico, nota-se a proliferação de adipócitos maduros, os quais estão separados por septos fibrosos, podendo apresentar diversos vasos sanguíneos e ser ou não envolto por uma cápsula de tecido conjuntivo fibroso (Neto et al., 2010; Ferreira et al., 2019). Os lipomas podem apresentar variantes como o fibrolipoma, lipomas mixóides, angiolipoma, lipoma de células fusiformes, lipomas pleomórficos, intramusculares e sialolipoma (Souza et al, 2010; Júnior et al., 2021). A excisão cirúrgica total da lesão é o tratamento mais indicado e, independentemente de sua variante histopatológica, as recidivas são raras (Resende; Meirelles; \& Varella, 2013; Osterne et al., 2019).

Neste contexto, o objetivo do presente estudo é apresentar um relato de caso de lipoma oral, enfatizando suas características clínicopatológicas e abordagem terapêutica.

\section{Metodologia}

Trata-se de um estudo de caso clinico qualitativo descritivo de um único paciente, onde foi relatado os métodos de diagnóstico e tratamento da neoplasia benigna em questão, o resultado deste tratamento e sua discussão com os achados na literatura. O paciente assinou o Termo de Consentimento Livre e Esclarecido (TCLE) e esteve ciente da divulgação das imagens clínicas (sem sua identificação) e dos procedimentos cirúrgicos realizados para enriquecimento da comunidade científica.

\section{Relato de Caso Clínico}

Paciente do sexo masculino, 56 anos, melanoderma, queixava-se de um "caroço na boca" com evolução de aproximadamente 4 anos e ausência de sintomatologia dolorosa. Ao exame intraoral foi observado um aumento de volume nodular, séssil, bem delimitado, com superfície íntegra e lisa, coloração amarelada, consistência amolecida, medindo aproximadamente $2,5 \mathrm{~cm}$ em seu maior diâmetro e localizado no fundo de vestíbulo entre os dentes 32 e 34 (Figura 1). 
Research, Society and Development, v. 10, n. 12, e325101219383, 2021

(CC BY 4.0) | ISSN 2525-3409 | DOI: http://dx.doi.org/10.33448/rsd-v10i12.19383

Figura 1 - Aspecto clínico da lesão. Nota-se presença de lesão nodular, bem delimitada, com superfície lisa, coloração levemente amarelada e localizada no fundo do vestíbulo entre dentes 32 e 34.

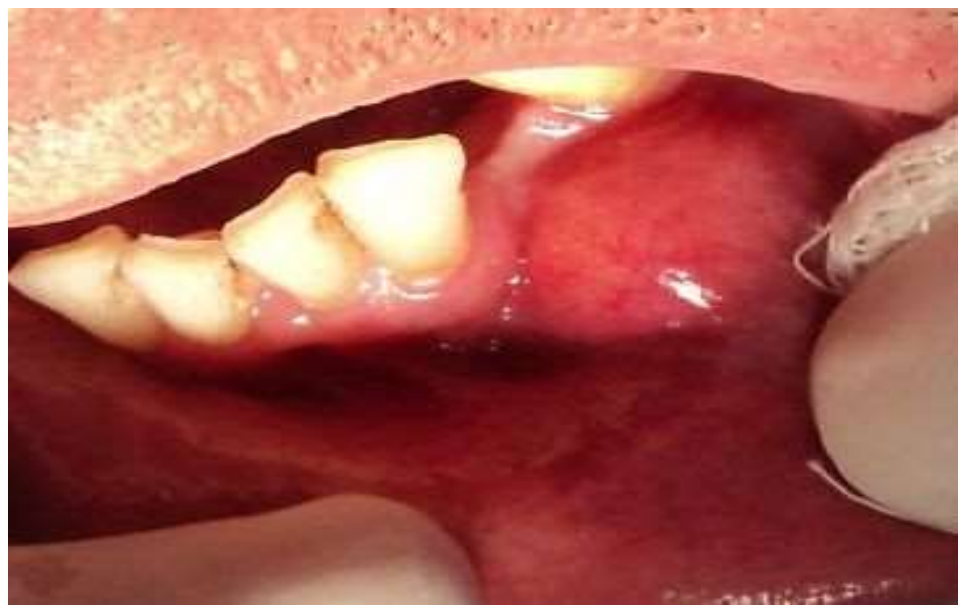

Fonte: Autores.

A lesão foi submetida à biópsia excisional, sem intercorrências $\mathrm{O}$ material removido foi encaminhado ao laboratório de histopatologia para confirmação diagnóstica (Figura 2A-D).

Figura 2: Biópsia excisional. A. Exposição cirúrgica da lesão. B. Espécime removido. C. Sítio cirúrgico com lesão removida.

\section{Sutura.}
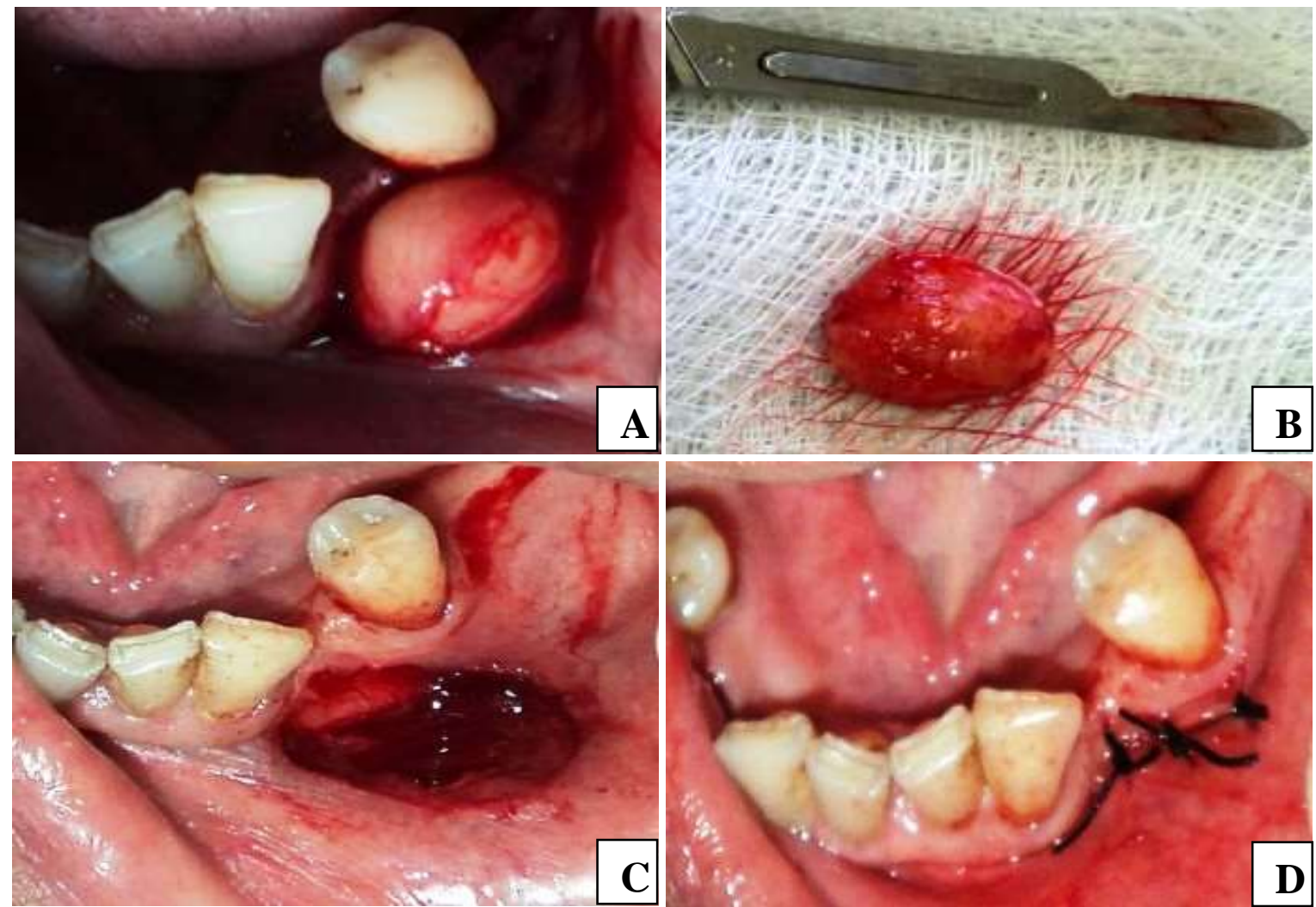

Fonte: Autores.

O exame histopatológico revelou a presença de neoplasia de origem mesenquimal composta por células adiposas 
maduras, organizadas em lóbulos separados por finos septos de tecido conjuntivo fibroso, os quais contêm vasos nutrientes, sendo o diagnóstico estabelecido como Lipoma. Paciente encontra-se há sete meses sem apresentar sinais de recidiva da lesão.

Figura 3: Análise histopatológica A. Neoplasia benigna com proliferação de adipócitos maduros delimitada por cápsula de tecido conjuntivo vascularizado. B. Adipócitos maduros com citoplasma amplo e claro.

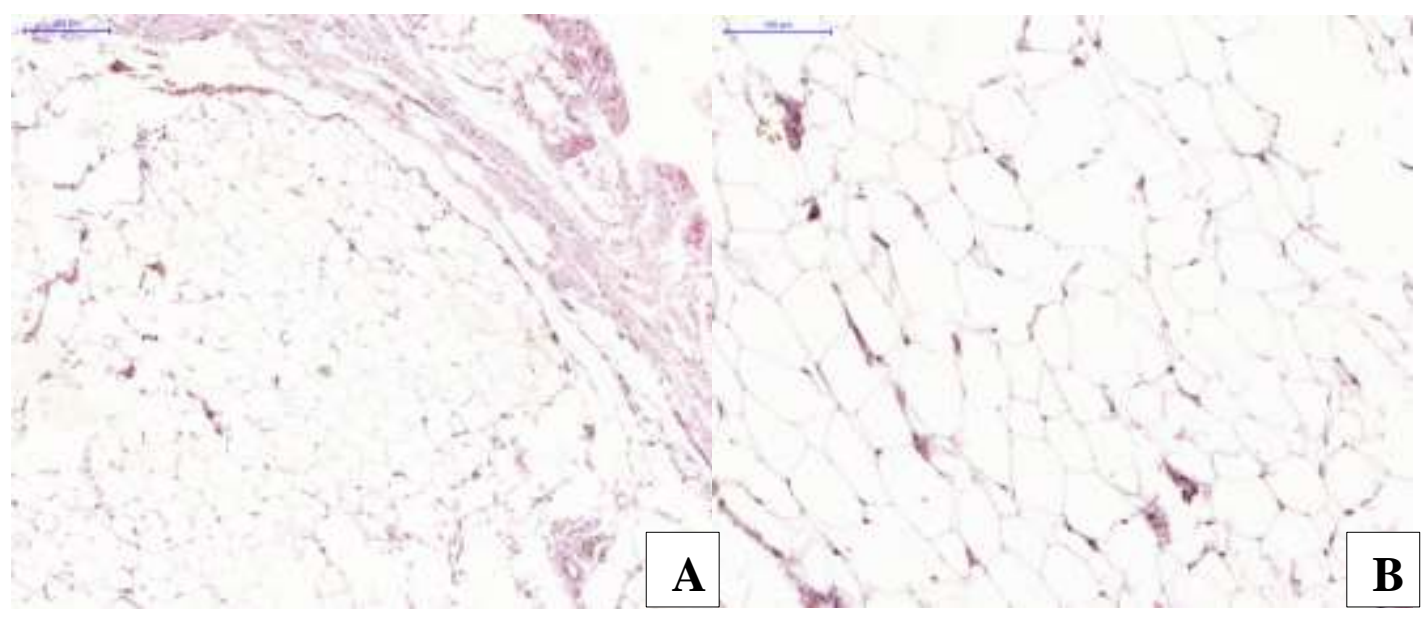

Fonte: Autores.

\section{Discussão}

Os lipomas são neoplasias mesenquimais benignas mais prevalentes, sendo comum em várias regiões do corpo como, por exemplo, tronco, ombros, pescoço e axila (Ponce et al., 2016; Linares et al., 2019). A cavidade oral representa apenas 1\% a 5\% da localização dos lipomas (Juliasse et al., 2010; Egido-Moreno et al., 2016). Apesar disso, ao realizarem um levantamento das neoplasias benignas diagnosticadas em um laboratório de Patologia oral, observaram que o lipoma é a quarta lesão mais frequente deste grupo, representando 10,2\% (Silva et al., 2017; Tenório et al., 2013).

Lipomas orais podem ser diagnosticados em indivíduos de qualquer faixa etária, porém os estudos revelam que o pico de incidência varia entre a $6^{\mathrm{a}}$ e $8^{\mathrm{a}}$ décadas de vida (Juliasse et al., 2010; Linares et al., 2019; Perez-Sayáns et al., 2019). A maioria das pesquisas mostra que esta neoplasia geralmente é mais comumente diagnosticada em mulheres, mas estes dados podem estar associados a fatores sociodemográficos das populações estudadas (Juliasse et al., 2010; Linares et al., 2019; PerezSayáns et al., 2019). No presente caso, a lesão foi diagnosticada em um paciente do sexo masculino na $6^{a}$ década de vida.

Clinicamente, os lipomas orais apresentam crescimento lento e assintomático, sendo estas características observadas, também, em casos de lipoma diagnosticado em outras regiões do corpo (Linares et al., 2019; Perez-Sayáns et al., 2019). No presente relato, o tempo decorrido entre o aparecimento do tumor (segundo informação do paciente) e a remoção cirúrgica foi de 4 anos, corroborando o estudo de Juliasse et al. (2010) que, ao avaliar 46 casos de lipomas, encontrou casos com tempo de duração variando entre 6 meses a 15 anos, tendo como média 4 anos. Na maioria dos casos, bem como o relatado neste estudo, o diagnóstico clínico do lipoma é facilitado devido a sua coloração tipicamente amarelada, entretanto, lesões localizadas em profundidade podem ser recobertas por espessa camada de tecido e, consequentemente, exibir coloração normal da mucosa. Outro achado característico da lesão é a flutuação no formol após sua remoção (Santos et al., 2017; Ferreira et al., 2019). Segundo os estudos de Dehghani et al (2019), o lipoma acaba sendo uma lesão de tecido mole bastante comum, mas raramente será encontrada em tecido duro, como é o caso do lipoma intra-ósseo na mandibula.

Lipomas orais geralmente apresentam, no momento do diagnóstico, tamanho médio de $2 \mathrm{~cm}$ (Naruse et al., 2015). No caso relatado neste estudo, a lesão mede $2,5 \mathrm{~cm}$ em seu maior diâmetro. Todavia, são relatados lipomas orais gigantes com até 
9cm (Chandak et al., 2012). A mucosa jugal é o sítio mais prevalente, totatalizando 37,5 a 53,7\% dos casos (Juliasse et al., 2010; Naruse et al., 2015; Linares et al., 2019). Essa predileção pode estar relacionada à disponibilidade de tecido adiposo, o qual é elevado nesta localização. Não existe consenso quanto ao segundo sítio mais acometido, com resultados variando entre língua e lábio, sendo o palato a região menos frequente (Perez-Sayáns et al., 2019). Aqui relatamos um caso diagnosticado em fundo de vestíbulo, local também caracterizado por apresentar considerável volume de tecido adiposo em condições de normalidade.

Histologicamente, grande parte dos lipomas orais apresenta em sua composição células adiposas maduras e organizadas em arranjo lobular (Vasconcelos et al., 2007; Júnior et al., 2021), características presentes no caso referido. Sabese que os lipomas apresentam ampla variedade de subtipos microscópicos e a sua prevalência varia em diferentes estudos. Todavia, é consenso que a variante clássica é a mais prevalente (Juliasse et al., 2010; Linares et al., 2019; Perez-Sayáns et al., 2019). O estudo de Osterne et al (2019) revela que 63,3\% dos casos foram classificados como a forma clássica do lipoma.

Como realizado no caso descrito, o tratamento mais recomendado para este tipo de tumor benigno consiste em uma excisão cirúrgica conservadora local. Quando removidos adequadamente apresentam baixas taxas de recidivas e, portanto, possuem um bom prognóstico (Ferreira et al., 2019; Boscaine et al, 2020). Nosso paciente encontra-se há 7 meses sem sinais de recidivas da lesão.

\section{Considerações Finais}

O conhecimento e a identificação do lipoma, bem como de todas as patologias encontradas na cavidade oral, são de responsabilidade do Cirurgião-Dentista. Cabe a este profissional, portanto, realizar um exame clínico preciso e intervir ou encaminhar adequadamente o paciente, portador da alteração, para que o mesmo seja adequadamente diagnosticado e tratado. Lipoma é uma neoplasia benigna, com crescimento lento e assintomático, que, assim como o presente caso, deve ser tratado cirurgicamente tendo baixas taxas de recidivas. $\mathrm{O}$ paciente do presente relato encontra-se há 7 meses sem sinais de recidiva da lesão.

\section{Referências}

Boscaine, E. F., Mendonça, J. C. G., Paiva-Oliveira, J. G., Pelissaro, G. S., Herculano, A. B. S., Figueiredo, F. T., Castillo,D. B., \& Gaetti-Jardim, E. C. (2020). Remoção cirúrgica de lipoma por acesso pré-auricular. Arch Health Invest, 9(6), 674-676. http://dx.doi.org/10.21270/archi.v9i6.4998.

Chandak, S., Pandilwar, P. K., Chandak, T., \& Mundhada R. (2012). Huge lipoma of tongue. Contemp Clin Dent, 3(4), 507-9. 10.4103/0976-237X.107457.

Dehghani, N., Razmara, F., Padeganeh, T., \& Mahmoudi, X. (2019). Oral lipoma: Case report and review of literature. Clinical case reports, 7(4), 809.

Ferreira, L. M., Rodrigues, R. D., Nogueira Neto, J. N., \& De Oliveira, J. F. C. D. (2019). Lipoma: relato de caso. Revista Odontológica de Araçatuba, 40(3), 24-27.

Juliasse, L. E. R., Nonaka, C. F. W., Pinto, L. P., Freitas, R. A., \& Miguel, M. C. C. (2010). Lipomas of the oral cavity: clinical and histopathologic study of 41 cases in a Brazilian population. Eur Arch Otorhinolaryngol, 267 (3), 459-465. 10.1007/s00405-009-1010-Z.

Júnior, L. G. S. D., Lima, V. B. S. R., Cabral, G. L., Azevedo, G. M. L., Júnior, J. R. S. S., Neto, J. C. S., \& Neves, R. F. S. N. (2021). Abordagem cirúrgica de lipoma intraoral em região de assoalho de boca à direita: relato de caso clínico. Research Society and Development, 10(1). http://dx.doi.org/10.33448/rsdv10i1.11509.

Lineares, F. M., Leonel, A. C. L. S., Carvalho, E. J. A., Castro, J. F. L., Almeida, O. P., \& Perez, D. E. C. (2019). Intraoral lipomas: A clinicopathological study of 43 cases, including four cases of spindle cell/pleomorphic subtype. Med Oral Patol Oral Cir Bucal, 24(3), e373-e378.

Naruse, T., Yanamoto, S., Yamada, S. I., Rokutanda, S., Kawakita, A., Takahashi, H., \& Yamashita, K. (2015). Lipomas of the oral cavity: clinicopathological and immunohistochemical study of 24 cases and review of the literature. Indian Journal of Otolaryngology and Head \& Neck Surgery, 67(1), 67-73.

Neto, N. R., Marques, J. A. F., Santos, M. A. M., Parra, G. R., Mota, G. C. C., \& Barreto, A. P. (2010). Lipoma of Incomun Size in Inferior Lip. Rev. cir. traumatol. buco-maxilo-fac.10(4).

Neville, B. W., \& Damm, D. D. (2016). Título: Patologia Oral \& Maxilofacial. 
Research, Society and Development, v. 10, n. 12, e325101219383, 2021

(CC BY 4.0) | ISSN 2525-3409 | DOI: http://dx.doi.org/10.33448/rsd-v10i12.19383

Osterne, R. L. V., Renata, M B., Lima-Verde, E., Nonaka, C. F. W., \& Cavalcante, R. B. (2019). Oral cavity lipoma: a study of 101 cases in a Brazilian population. Jornal Brasileiro de Patologia e Medicina Laboratorial, 55(2), 148-159. https://doi.org/10.5935/1676-2444.20190017.

Perez-Sayáns, M., Blanco-Carrión, A., Oliveira-Alves, M. G., Almeida, J. D., Anbinder, A. L., Mendoza, I. L. I., \& Aguirre-Urízar, J. M. (2019). Multicentre retrospective study of 97 cases of intraoral lipoma. Journal of Oral Pathology and Medicine, 48(6), 499-504. http://dx.doi.org/10.1111/jop.12859.

Ponce, J. B., Ferreira, G. Z., Santos, P. S. S., \& Lara, V. S. (2016). Giant oral lipoma: a rare entity. An. Bras. Dermatol., Rio de Janeiro, 91(5), supl. 1, 84-86. https://doi.org/10.1590/abd1806-4841.20165008.

Resende, R., Meirelles, M., \& Varella. (2013). Remoção cirúrgica de lipoma de grande proporção: Relato de caso. Rev. cir. traumatol. buco-maxilo-fac., 13(2).

Santos, L. A. M., Barbalho, J. C. M., Costa, D. F. N., Silva, C. C. G., Pereira, V. B. S., \& Vasconcelos, B. E. C. (2014). Lipoma intraoral: relato de caso. Rev. cir. traumatol. buco-maxilo-fac., 14(3).

Souza, C. G., Souza, D. O., Nunes, T. S., \& Araujo, R. P. C. (2010). Tratamento de múltiplos lipomas de língua: relato de caso clínico. Revista de Ciências Médicas e Biológicas, 9 (Supl.1), 104-107.

Souza, F. R. N., CastroI, A. L., Moraes, N. P., Soubhia, A. M. P., Júnior, E. G. J., \& Miyahara, G. I. (2008). Lipoma em mucosa bucal. Rev. Cir. Traumatol. Buco-Maxilo-fac., Camaragibe, 8(3), 31-34.

Valencia, P. E. M., Rivera, V. A. B., \& Mazuera, L. M. R. (2017). LIPOMA GINGIVAL: UM RELATO DE CASO. Rev Fac Odontol Univ Antioq., 29(1). https://doi.org/10.17533/udea.rfo.v29n1a11.

Vasconcelos, B. C. E., Porto, G. G., Carneiro, S. C. A., \& Xavier, R. L. F. (2007). Lipomas da cavidade oral. Rev. Bras. Otorrinolaringol., 73 (6), https://doi.org/10.1590/S0034-72992007000600017.

Tenório, J. D. R., Paiva, K. M. D., Nogueira, P. T. B. D. C., \& Silva, E. D. D. O. (2013). Exérese de extenso lipoma em região submandibular: relato de caso. Revista de Cirurgia e Traumatologia Buco-maxilo-facial,13 (3), 43 - 50. 